\title{
Genome-wide dynamic network analysis reveals the potential genes for MeJA- induced growth-to-defense transition
}

Tengfei Wang ${ }^{1,2,3}$ and Xiujun Zhang ${ }^{1,2^{*}}$

\begin{abstract}
Background: Methyl jasmonate (MeJA), which has been identified as a lipid-derived stress hormone, mediates plant resistance to biotic/abiotic stress. Understanding MeJA-induced plant defense provides insight into how they responding to environmental stimuli.

Result: In this work, the dynamic network analysis method was used to quantitatively identify the tipping point of growth-to-defense transition and detect the associated genes. As a result, 146 genes were detected as dynamic network biomarker (DNB) members and the critical defense transition was identified based on dense time-series RNA-seq data of MeJA-treated Arabidopsis thaliana. The GO functional analysis showed that these DNB genes were significantly enriched in defense terms. The network analysis between DNB genes and differentially expressed genes showed that the hub genes including SYP121, SYP122, WRKY33 and MPK11 play a vital role in plant growthto-defense transition.
\end{abstract}

Conclusions: Based on the dynamic network analysis of MeJA-induced plant resistance, we provide an important guideline for understanding the growth-to-defense transition of plants' response to environment stimuli. This study also provides a database with the key genes of plant defense induced by MeJA.

Keywords: Methyl jasmonate (MeJA), Growth-to-defense transition, Plant resistance, Dynamic network biomarker (DNB), Tipping point

\section{Introduction}

In response to changing environments, plants have to manage their resources to reach a balance between growth and defense in order to survive and reproduce $[1,2]$. Rapid growth and accumulation of nutrients help plants compete for more resources. While an effective and inducible defense system at the cost of resources has evolved in plants to resist biotic/abiotic stress [3, 4].

\footnotetext{
*Correspondence: zhangxj@wbgcas.cn

${ }^{1}$ CAS Key Laboratory of Plant Germplasm Enhancement and Specialty

Agriculture, Wuhan Botanical Garden, Chinese Academy of Sciences, 430074 Wuhan, China

${ }^{2}$ Center of Economic Botany, Core Botanical Gardens, Chinese Academy of Sciences, 430074 Wuhan, China

Full list of author information is available at the end of the article
}

The correct and effective transition from growth to defense is crucial for plants' survival and wellbeing [5, 6]. Several studies have correlated some phytohormones with the regulation of plant defense and identified multiple factors as the participants of the pathway [7-9]. Revealing the basic mechanism of hormone-induced growth-to-defense transition is essential to elucidate how plants respond to environmental stimuli $[10,11]$ and carry important implications to ecological and agricultural improvement $[12,13]$.

Methyl jasmonate (MeJA) and its free-acid jasmonic acid (JA) were well-recognized as lipid-derived stress hormones $[14,15]$. JAs-mediated growth-to-defense tradeoffs regulate plant defense against necrotrophic pathogens and insect herbivores $[16,17]$. In previous studies,

(c) The Author(s). 2021 Open Access This article is licensed under a Creative Commons Attribution 4.0 International License, which permits use, sharing, adaptation, distribution and reproduction in any medium or format, as long as you give appropriate credit to the original author(s) and the source, provide a link to the Creative Commons licence, and indicate if changes were made. The images or other third party material in this article are included in the article's Creative Commons licence, unless indicated otherwise in a credit line to the material. If material is not included in the article's Creative Commons licence and your intended use is not permitted by statutory regulation or exceeds the permitted use, you will need to obtain permission directly from the copyright holder. To view a copy of this licence, visit http://creativecommons.org/licenses/by/4.0/ The Creative Commons Public Domain Dedication waiver (http://creativecommons.org/publicdomain/zero/1.0/) applies to the data made available in this article, unless otherwise stated in a credit line to the data. 
many genes of the JA signaling pathway were identified as participants of plant defense [18-20]. By the crosstalk with other phytohormones, JAs take part in the defense against abiotic stresses and biotic stresses [21-23]. These results indicated that JAs-induced growth-to-defense transition plays a crucial role in plant defense against the changing environments $[24,25]$.

To understand the molecular mechanism of plants' defense induced by JAs, some genetic, molecular, and physiological approaches have been used and some results were achieved. The core JA signaling pathway was discovered as a co-receptor complex consisting of the F-box protein CORONATINE INESENSTIVE 1 (COI1) and the JASMONATE ZIM DOMAIN (JAZ) family of transcription repressors. The COI1-JAZ complex perceives jasmonoyl-1isoleucine (JA-Ile), leading to poly-ubiquitylation of JAZ and its subsequent degradation. The degradation of JAZ relieves the repression of JAZ-interacting transcription factors, permitting the expression of related genes and execution of physiological responses, with multiple feedback loops to ensure timely termination of these responses $[18,26]$.

Network-based analysis methods have been developed to identify candidate genes and elucidate the molecular mechanisms of complex biological processes at a system level $[27,28]$. Among the network inference methods, mutual information-based methods enable accurate quantification of associations in gene regulatory networks $[29,30]$. The weighted correlation network analysis (WGCNA) has been widely used to detect gene modules that were related to some special traits [31]. In recent studies, network-based analysis methods have also been used to investigate the mechanism of growthdefense tradeoffs under stress or defense signals in plants [32, 33].

In recent years, the phase transition of disease development has been studied, such as the pre-disease state of diabetes mellitus and the critical transition of breast cancer [34, 35]. For plants, our previous work has studied the state transition of flowering and some genes were identified as the controllers of flowering [36]. The transition of complex system is usually non-smooth or abrupt in many biological processes [37]. The plant system in growth or defense state is stable and highly resilient. Plant usually survives by balancing growth and defense, i.e. it accumulates nutrients by former whereas costs resources by latter [1].

During the balance between plant growth and defense, the tipping point is the critical transition point of the transition of the system from growth to defense. The tipping point is not only a time point but also a response process of the system. At the tipping point, the expressions of the related genes fluctuate drastically. The unstable state also leads to increased fluctuation in the observed transcript expression level. In transition state, the violent fluctuations within the plant system include the transmission of defense signals, the redistribution of metabolic pathways, and the reallocation of resources. Identifying the critical state or tipping point of growthto-defense transition is important to understand the systemic defense in plants. The system at the tipping point is fragile and susceptible to change. Therefore, the tipping point-associated genes are vital to the defense system $[1,2]$.

The time-series high-throughput sequencing data provide an unprecedented opportunity to study the mechanism of plant systemic resistance at the genome-wide scale [38, 39]. Here, a dynamic network analysis method called DNB, which was designed to quantitatively identify the tipping point of a drastic system transition, can theoretically identify a group of highly correlated and strongly fluctuated genes [34, 37].

In this work, we applied the DNB method to detect the critical point of plant grow-to-defense transition and identify plant resistance-associated genes. As a result, 146 genes were detected as DNB members and the 11th time $(8 \mathrm{~h})$ period was identified as a critical transition point based on time-series high-throughput RNA-seq data of MeJA-treated Arabidopsis thaliana. Among the DNB genes, many of them have been proved to be widely involved in plant defense. The GO functional enrichment analysis showed that these DNB genes were significantly enriched in defense terms. The STRING network with 287 nodes and 807 edges confirmed the close association between the DNB genes and the differentially expressed genes. The network analysis also showed that the hub genes including SYP121, SYP122, WRKY33, MPK11 and at5g22920 play a vital role in plant growth-to-defense transition. Based on the dynamic network analysis of MeJA-induced plant resistance, we provide an important guideline in understanding the growth-defense transition of plant responding to environment stimuli. We also provide a database with the key genes of plant defense induced by MeJA.

\section{Results \\ Composite index for detecting the tipping point of growth-defense transition}

The transition of complex system is usually nonsmooth or abrupt in many biological processes. Such a transition changes the state of the biological system qualitatively and thus plays key roles in biological processes. The process of plant from rapid growth and accumulation of nutrients to depletion of resources for defense can be divided into three stages, i.e. growth state, critical transition state and defense state (Fig. 1a). 
(a)

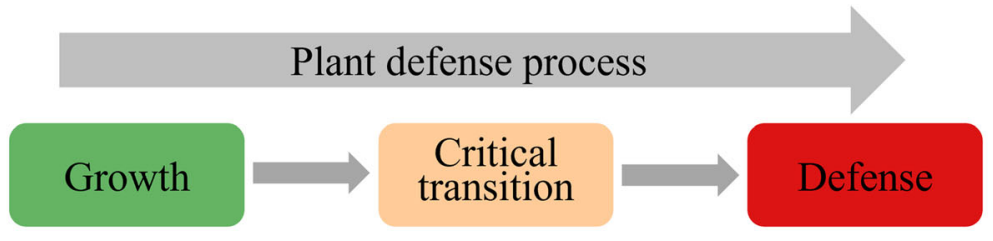

(b)

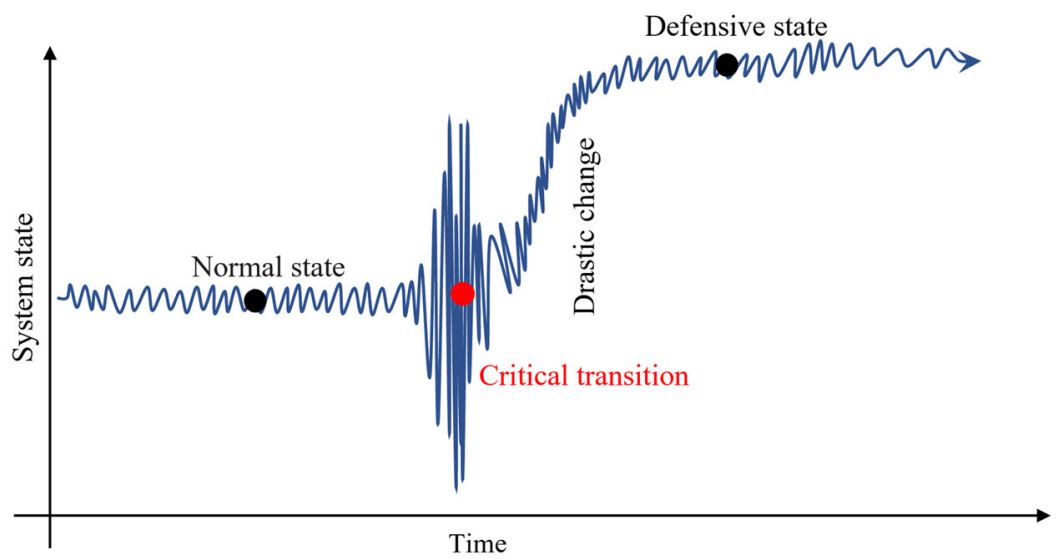

(c)
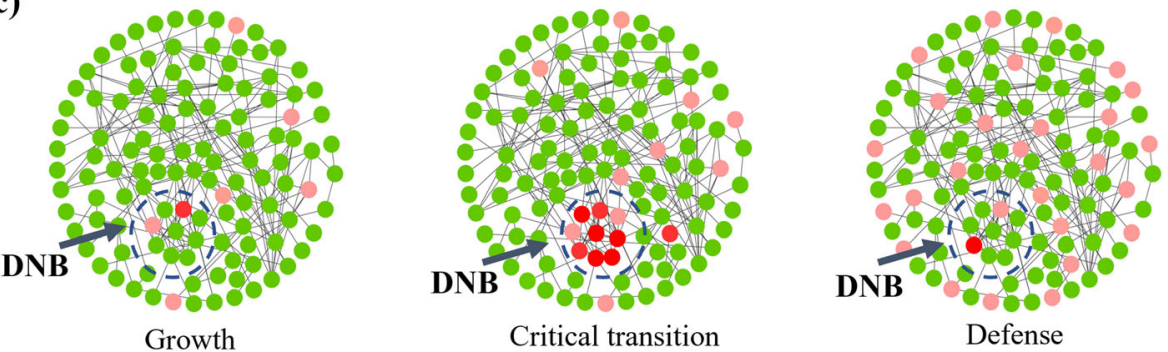

Fig. 1 The schematic diagram for the identification of the critical point of plant growth-defense transition by dynamic network analysis. a The plant defense process from rapid growth to depletion of resources for defense can be divided into three states: growth state, critical transition state and defense state. $\mathbf{b}$ The system in growth or defense state is stable and resilient. In transition stage, the violent fluctuations within the plant system include the transmission of defense signals, the dramatic fluctuations in gene expression, and the redistribution of metabolic pathways. c In the critical transition state, the deviations of DNB members increase sharply and the correlations between DNB members increase obviously

The system in growth or defense state is stable and highly resilient. In transition state, the violent fluctuations within the plant system include the transmission of defense signals, the dramatic fluctuations in gene expression, and the redistribution of metabolic pathways (Fig. 1b). To identify the tipping point of defense transition and understand the molecular mechanism of growth-defense tradeoffs, the dynamic network analysis method (DNB, dynamic network biomarkers) was used. The DNB method can detect critical transition state from the measured data based on nonlinear dynamic theory, which has been theoretically and numerically proved. A system is near to the critical transition state or tipping point if there is a dominant group of molecules or genes satisfying the following three criteria from the observed data: (1) Standard deviations of genes in this dominant group (denoted by $S D i$ ) increased markedly; (2) Pearson correlation coefficients between genes in this dominant group (denoted by $P C C i$ ) increased significantly; (3) Pearson correlation coefficients between genes in the group and genes outside of the group (denoted by $P C C o$ ) decreased significantly. The following composite index $(\mathrm{CI})$ approximately considering all three criteria can be used as the numerical signal of the DNB method:

$$
C I=S D i * P C C i / P C C o,
$$

where $S D i$ is the average standard deviation of all genes in the dominant group, $P C C i$ is the average PCC of all gene-pairs in the dominant group (absolute value), and PCCo is the average PCC of gene-pairs between the dominant group and others (absolute value). The definition of CI shows that there are a group of genes fluctuating strongly in the critical transition state. The group members are the dynamic network biomarkers of the 
critical transition. When $C I$ reaches a peak or increases markedly during the periods, the biological system is at the critical transition state or tipping point (Fig. 1c).

In contrast to the traditional differential expression analysis, the DNB method considered both molecular fluctuation information (dynamic information) and network information (correlation information among genes).

Dynamic network analysis of MeJA-induced plant defense DNB is a method to identify gene module for the transition. In a DNB module, the standard deviations of genes increase markedly, the correlations of genes increase significantly while the correlations between genes in and out of the module decrease significantly. The procedure for the dynamic network analysis was described in Fig. 2. The time-series high-throughput sequencing data was aligned and assembled to obtain gene expression matrix for further dynamic network analysis (Fig. 2a).

The dynamic network analysis of plant growth-defense transition was performed with the following steps. Firstly, hierarchical clustering was implemented on the gene expression data to obtain candidate gene modules for each time point (Fig. 2a, b). Secondly, the CIs of candidate modules were calculated and the maximum $\mathrm{CI}$ (noted as CI_max) was selected for each time point. The selected CI_maxs for all the time points were compared to detect the tipping (critical transition) point. The time point with highest CI_max was considered as the critical transition point (Fig. 2b, c). Thirdly, at the detected critical transition point, the module with the highest CI_max was recognized as DNB module (Fig. 2d). Lastly, the Arabidopsis thaliana protein-protein interactions (PPIs) in STRING database were used as background network (Fig. 2e). The core network for plant growth-defense transition was constructed from DEGs directly interacted with DNB genes in background network (Fig. 2d, f).

\section{Tipping point of plant defense identified by DNB}

The time-series RNA-seq dataset of MeJA-treated Arabidopsis thaliana at 13 time points was analyzed to detect the critical transition of plant growth-to-defense and identify the associated genes. As is shown in Fig. 3, the 11th time point ( $8 \mathrm{~h}$ after MeJA treatment) with the highest CI_max value (3.03) in all modules was identified as the critical transition state of plant defense and marked in red.

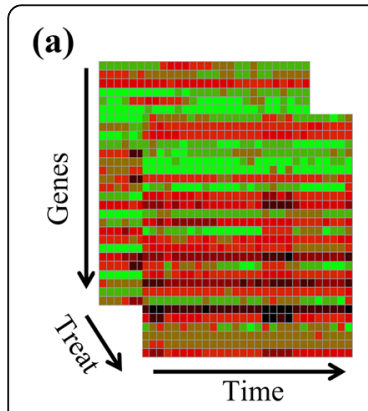

Gene expression data

(d)

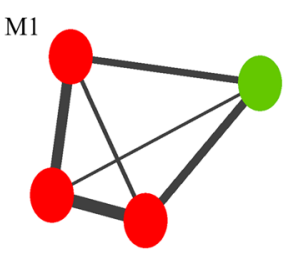

DNB module (b)
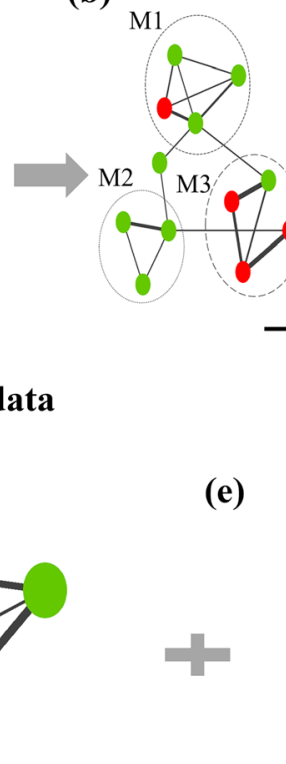

Gene modules

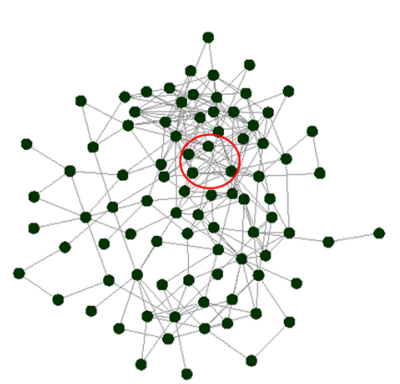

Background network

(c)

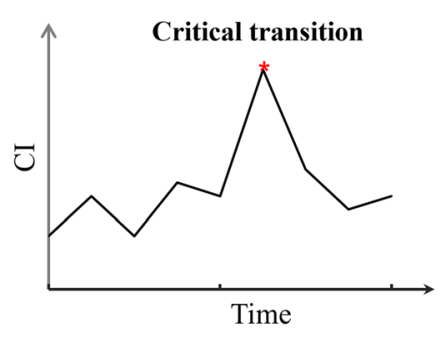

DNB score

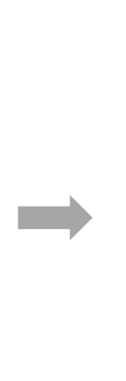

(f)

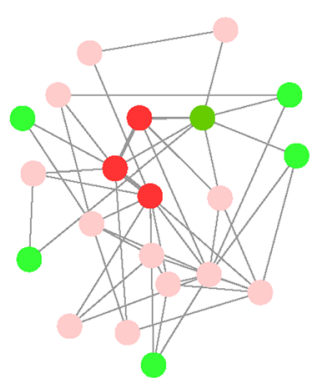

Core network

Fig. 2 Flowchart of dynamic network analysis of MeJA-induced plant defense. a Control and MeJA-treated time-series gene expression data were normalized. b Gene modules (e.g., M1, M2, and M3) were constructed based on Pearson correlation coefficients between genes. Genes with standard deviation (SD) increased sharply are shown in red, others are in green. c Cl scores were calculated to quantify DNB module. The time point with the highest $\mathrm{Cl}$ was detected as the tipping point of transition (critical transition state), marked in red. $\mathbf{d}$ DNB modules were inferred from the candidate of gene modules. The module of genes with the highest Cl_max was recognized as DNB module. e Protein-protein interactions (PPIS) from STRING network. The high confidence of interactions was used as background network for DNB genes. $\mathbf{f}$ Core network for plant defense were constructed based on DNB genes and background network 


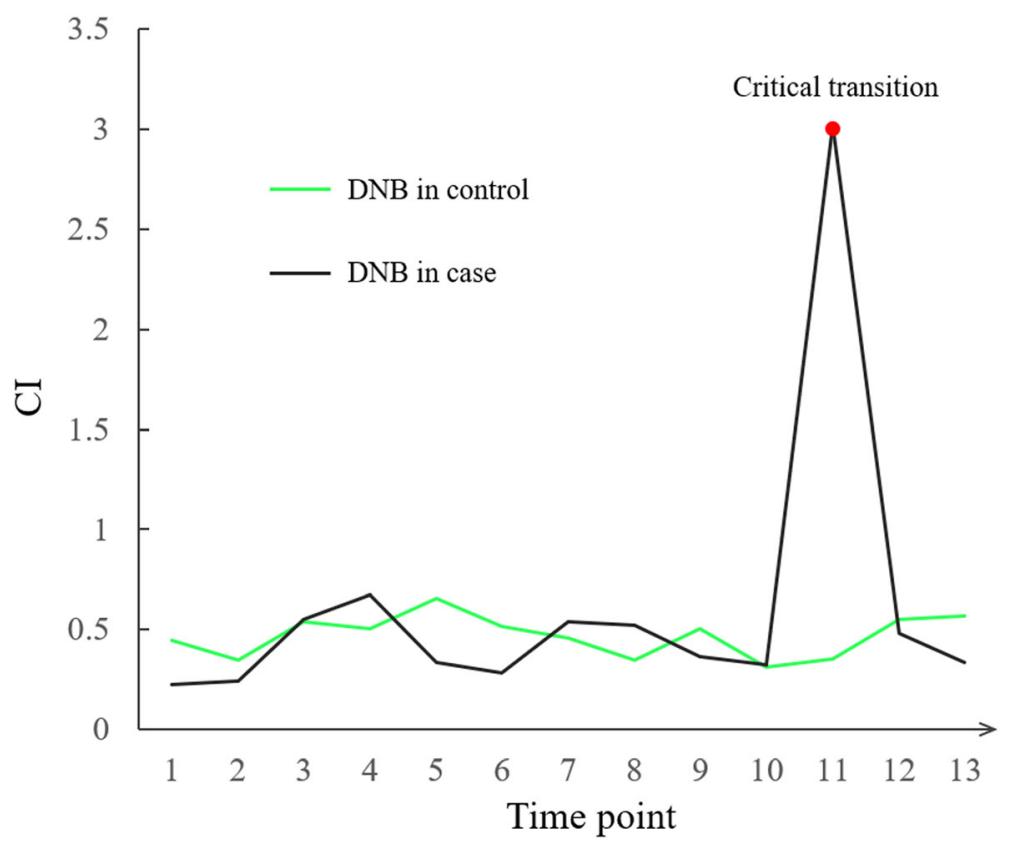

Fig. 3 The Cl of DNB module in different periods. The Cl of DNB module was marked in black in data from case samples (MeJA treated) and was marked in green in data from control samples (MOCK treated). The highest $\mathrm{Cl}$ score for DNB module marked as a red dot at point 11

By analyzing the CIs of DNB modules, we found that CI reached highest at the tipping point. At the critical transition state, the average standard deviation (SD) of DNB members reached the maximum, the correlation increased significantly and the correlation outside the DNB module was lowest (Supplementary figure S1). The dynamics of SD showed that the expression of DNB genes was highly fluctuating at the tipping point. The correlation analysis of genes in and out of the DNB module showed that the DNB genes were highly correlated with the genes in the module while weakly correlated with the genes outside of the module (Supplementary Figure S1). This result showed that the emergence of such a cluster of genes approved it a signal of developmental state transition.

To verify the biological and statistical significance of the identified DNB module, the bootstrap analysis was performed. The result showed that it was significant for the CIs of DNB modules compared with the CIs of random gene modules (Supplementary Figure S2).

Identified DNB genes are widely involved in plant defense As another result of the dynamic network analysis, a DNB module with 146 genes was identified as the key genes for plant growth-to-defense transition. With the gene function annotation, we found that that the genes identified by DNB were widely involved in the initiation of plant defense and some of them played key roles in the regulation of plant defense. For instance, as a central metabolic regulator, transcription factor bZIP63 is essential for stress responses [40, 41]. Another ZIP transcription factor HAT22 regulates a set of genes implicated in stress responses and restricts new shoot growth [42]. Gene MYB3R5 with repressor function restrict the expression of gene with mitotic functions to inhibit plant growth [43]. Gene TOPP9 encodes Type One Protein Phosphatase that acts as a nucleocytoplasmic negative regulator of tip growth and affects the mitogen-activated protein kinases (MAPKs)-mediated downstream defense pathway [44]. It is a remarkable note that the DNB method can identify genes that play a key role in function but not show significant differential expression.

The differential expression analysis of DNB genes was performed between the critical transition points $(8 \mathrm{~h}$ after MeJA treated) and the previous point $(7 \mathrm{~h}$ after MeJA treated). Table 1 showed the functional description and expression changes of the DNB genes which were crucial for defense transition. The genes with fold change $(\mathrm{FC})>1.5$ and false discovery rate $(\mathrm{FDR})<0.05$ were selected as DEGs. Moreover, all the DNB genes with gene description were shown in Supplementary Table S1.

\section{Function analysis for DNB genes}

The Gene Ontology (GO) term enrichment analysis was implemented to classify the function of DNB genes at the critical transition of Arabidopsis thaliana induced by MeJA. In the biological process category, 'response to chitin' and 'response to other organonitrogen 
Table. 1 Functional description and expression changes of the DNB genes which crucial for defense transition

\begin{tabular}{|c|c|c|}
\hline $\begin{array}{l}\text { DNB } \\
\text { Genes }\end{array}$ & $\begin{array}{l}\text { Fold } \\
\text { Change }\end{array}$ & Gene Description \\
\hline \multicolumn{3}{|c|}{ Transcription factor } \\
\hline bZIP63 & $25.81^{b}$ & $\begin{array}{l}\text { BASIC LEUCINE ZIPPER protein which regulates the circadian oscillator gene PRR7 to change the circadian phase in } \\
\text { response to sugars. }\end{array}$ \\
\hline HAT22 & $2.34^{\mathrm{a}}$ & Encodes homeobox protein HAT22, member of the HD-Zip II family. The mRNA is cell-to-cell mobile. \\
\hline ERF043 & $3.33^{\mathrm{a}}$ & Encodes a member of the DREB subfamily A-4 of ERF/AP2 transcription factor family. \\
\hline CRF6 & $4.15^{\mathrm{a}}$ & CRF6 encodes one of the six cytokinin response factors. \\
\hline $\mathrm{BPC1}$ & 2.83 & BASIC PENTACYSTEINE1 (BPC1) is a regulator of the homeotic Arabidopsis thaliana gene SEEDSTICK (STK). \\
\hline MYB3R-5 & 1.65 & Encodes a putative c-MYB-like transcription factor of the MYB3R factor gene family. \\
\hline AT5G26865 & 1.27 & AGAMOUS-like MADS-box protein \\
\hline AT3G58630 & 1.08 & sequence-specific DNA binding transcription factor \\
\hline
\end{tabular}

\section{Response to stress}

$\mathrm{TCH} 3.68^{\circ}$

ERD15 $\quad 3.77^{b}$

TMAC2 $21.70^{b}$

AGB1 $\quad 1.78^{b}$

AT1G03610 $2.95^{b}$

CINV1 $\quad 1.85^{b}$

PP2-A13 $\quad 4.52^{b}$

AT4G26750 $1.87^{\mathrm{b}}$

PLIP2 $\quad 9.32^{b}$

SAP2 $\quad 4.65^{b}$

KPI-1 $2.11^{a}$

ACBP3 $\quad 5.07^{\mathrm{a}}$

$\begin{array}{ll}\text { SYP121 } & 2.19\end{array}$

ERD14 1.52

MBL $1 \quad 6.81$

AT1G19310 1.51

SAG21 $\quad 3.18$

SGT1A $\quad 1.39$

ASAP1 $\quad 5.62$

EAP3 $\quad 1.80$

RMA1 1.98

SYP42 1.63

$\begin{array}{ll}\text { NTAQ1 } & 0.77\end{array}$
UGT76C2 1.78

Encodes a calmodulin-like protein, with six potential calcium binding domains. Expression may also be developmentally controlled.

Encodes hydrophilic protein lacking Cys residues that is expressed in response to drought stress, light stress and treatment with plant-growth-promoting rhizobacteria.

Encodes a protein that acts in the nucleus and is an important negative regulator of ABA and salt stress responses.

Encodes the heterotrimeric G-protein beta subunit and is involved in organ shape. A significant fraction of the protein is found in the ER.

plant/protein (DUF789)

CINV1 is an alkaline/neutral invertase that breaks sucrose down into fructose and glucose (GH100).

phloem protein 2-A13

Encodes a protein involved in mediating plant responses to pathogenesis.

PLIP2 is a glycerolipid A1 lipase with substrate preference for monogalactosyldiacylglycerol. Expression is induced by ABA.

Eukaryotic aspartyl protease family protein

Encodes a Kazal-type serine proteinase inhibitor that is highly expressed in seedlings and flowers.

acyl-CoA-binding protein ACBP3. Shows up-regulation of many biotic and abiotic stress related genes.

Encodes a syntaxin localized at the plasma membrane. SYR121/PEN1 is a member of the SNARE superfamily and functions in positioning anchoring of the KAT1 $\mathrm{K}+$ channel protein at the plasma membrane.

Encodes a dehydrin protein whose expression is induced early on in response to dehydration stress.

curculin-like (mannose-binding) lectin family protein, low similarity to ser/thr protein kinase from Zea mays; contains Pfam lectin (probable mannose binding) domain PF01453 but not the protein kinase domain of the Z. mays protein.

RING/U-box superfamily protein

Encodes a cytokinin N-glucosyltransferase that is involved in cytokinin homeostasis and cytokinin. Expression is induced by $A B A$, mannitol and drought stress.

Encodes AtLEA5 (late embryogenesis abundant like protein). Also known as SENESCENCE-ASSOCIATED GENE 21 (SAG21). Elevated in response to various stresses.

Closely related to SGT1B, may function in SCF(TIR1) mediated protein degradation. AtSGT1a and AtSGT1b are functionally redundant in the resistance to pathogenes.

NSE5 subunit of the SMC5/6 complex.

EAP3 is a cytolsolic BTB/POZ-domain protein involved in trafficking of PEN3.

RMA1 encodes a novel $28 \mathrm{kDa}$ protein with a RING finger motif and a C-terminal membrane-anchoring domain that is involved in the secretory pathway.

Encodes a member of SYP4 Gene Family that is a plant ortholog of the Tlg2/syntaxin16 Qa-SNARE. Together with SYP43, it regulates the secretory and vacuolar transport pathways in the post-Golgi network.

Controls the expression of specific defence-response genes, activates the synthesis pathway for the phytoalexin camalexin and influences basal resistance to Pseudomonas syringae pv tomato (Pst).

\section{Negative regulation of growth}

TOPP9 $\quad 4.35^{\mathrm{b}} \quad$ Encodes a Type One Protein Phosphatase that acts as a nucleocytoplasmic negative regulator of tip growth. 
Table. 1 Functional description and expression changes of the DNB genes which crucial for defense transition (Continued)

\begin{tabular}{lll}
\hline $\begin{array}{l}\text { DNB } \\
\text { Genes }\end{array}$ & $\begin{array}{l}\text { Fold } \\
\text { Change }\end{array}$ & Gene Description \\
\hline CDPK2 & $7.21^{\mathrm{b}}$ & $\begin{array}{l}\text { Encodes a Ca(2+)-dependent, calmodulin-independent protein kinase that is rapidly induced by drought and high-salt } \\
\text { stress. Positive regulator of ABA signaling. }\end{array}$ \\
\hline
\end{tabular}

${ }^{a}$ indicates significance at 0.05 level $^{\mathrm{b}}$ indicates significance at the 0.01 level

compound' were most common GO terms for the DNB genes. Some other significantly enriched GO terms include 'response to unfolded protein', 'regulation of innate immune response', and so on (Fig. 4a). In the molecular function category, these GO terms were enriched in DNB genes, such as 'arsenate reductase (glutaredoxin) activity', 'oxidoreductase activity', 'acting on phosphorus or arsenic in donors', 'disulfide as acceptor', 'SNAP receptor activity', and 'beta-fructofuranosidase activity' (Fig. 4b). None GO term was enriched in the cellular component category. All the enriched GO terms of DNB genes in the two GO categories (biological process and molecular function) were listed in Supplementary Table S2.

The Kyoto Encyclopedia of Genes and Genomes (KEGG) metabolic pathway analysis was implemented for checking the additional functional information of these identified genes [45]. These DNB genes were assigned to the reference pathway in KEGG to further check the biological function. As a result, 30 genes were mapped to about 26 pathways. The 'plant-pathogen interaction' pathway is the most common among these pathways. Besides, genes ARR4 and PYL5 were found to be involved in 'plant hormone signal transduction', genes
PGL1 and GDH2 were found to be involved in 'carbon metabolism' pathway, and genes SYP121 and SYP42 were found to be involved in 'SNARE interactions in vesicular transport' pathway. The DNB genes assigned KEGG pathways were shown in Supplementary Table S3. The GO and KEGG analyses revealed that DNB genes were closely related to plant defense and signaling pathway.

\section{Core network of DNB genes and differentially expressed genes}

To discover the associations between dynamic network biomarker (DNB) genes and differentially expressed genes (DEGs), the differential gene expression analysis was performed for defense tipping point and previous time. The result showed that 1434 genes (Supplementary Table S4) were differentially expressed between the defense tipping point and previous time point. Among these genes, 991 genes were up-regulated and 443 genes were down-regulated (Fig. 5a). Subsequently, a core network was constructed consisting of 287 nodes with 708 edges between DNB genes and DEGs based on the STRING network (Fig. 5). In this network, the red nodes represented DNB genes, green nodes represented down-

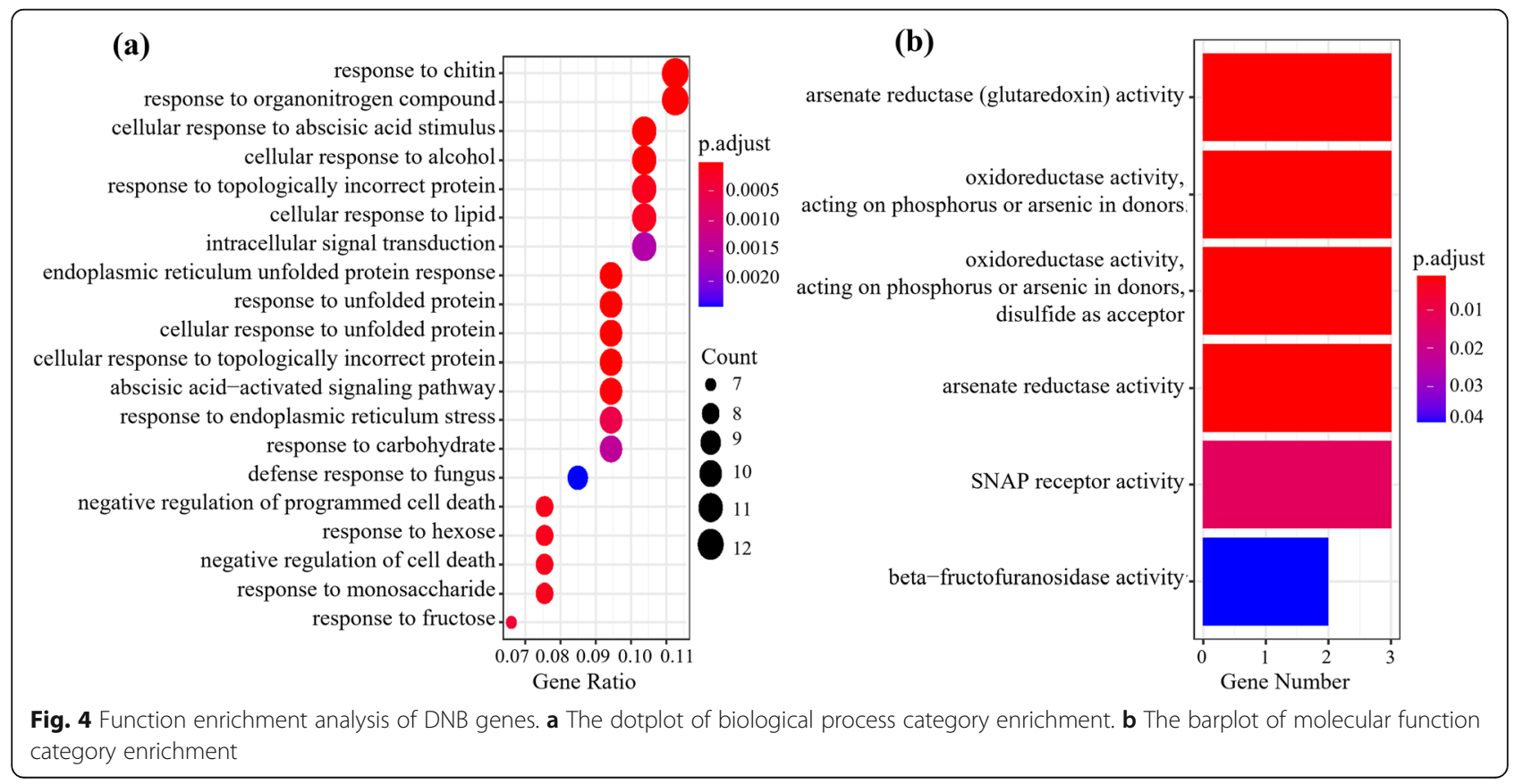


regulated DEGs, and pink nodes represented the upregulated DEGs. The network of DNB genes and DEGs was shown in Supplementary Table S5.

In addition, we analyzed the function of hub genes of the core network in plant growth-to-defense transition. The result showed that 7 out of the top 10 hub genes including SYP121, SYP122, WRKY33, NHL3, TZF11, and AT1G19020 play a key role in the regulation of plant resistance. The functional descriptions of the top 10 hub genes were shown in Supplementary Table S6.

\section{Discussion}

The growth-defense transition is the form of plant responding to (a)biotic stress or defending signals. This method is an effective approach to study crop improvement by identifying the genes that control the growthdefense transition [46-48]. With the function of rewiring central metabolism to promote defense and inhibit growth, JAs play a key role in growth and defense tradeoffs [24, 26, 49]. Some genes related to growth-defense transition have been found in the JAs pathway $[48,50]$.

The high-resolution time-series RNA-seq data provide the opportunity to identify the critical state or tipping point of plant growth-defense transition [51]. At the tipping point, the JA response genes in format of a module fluctuate drastically in expression. For example, the expression of these genes decreased in plant growth phase while increased in defense phase. Subsequently, the plant minimizes growth and initiated defense. These genes were taken as the key factors for the growth-defense transition [52]. In our opinion, the tipping point is the transition state of plant growth-defense transitions in theory. For the result of data analysis, the detected tipping point was just the nearest one to the transition state among all the time points measured for the timeintensive data. The methods for identifying key genes that involve in the JA gene regulatory network are important for understanding of plant growth-defense transitions [38]. To understand the mechanism of plant growth-defense transitions, the special methods which can accurately model JA defend response should be developed [32].

To investigate the JA gene regulatory network, highresolution time series RNA-seq for MeJA-treated Arabidopsis thaliana were generated [51]. With computational analysis, this study provided insight into the dynamics and architecture of the JA gene regulatory network. Several TFs including MYB59 and bHLH27 were uncovered as early network components with a role in pathogen and insect resistance. What's more, this (a)

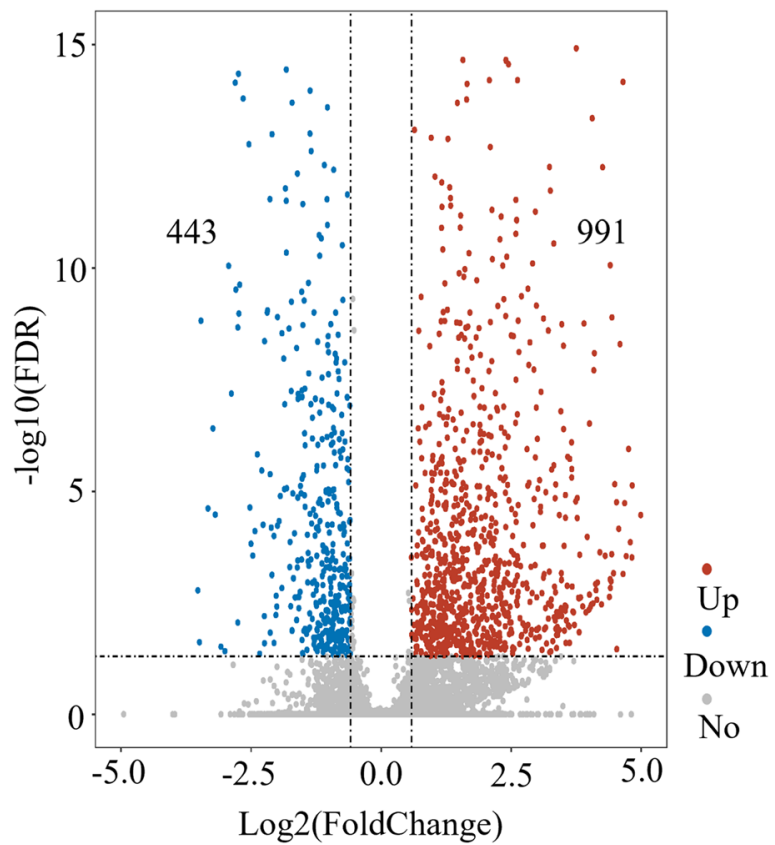

(b)

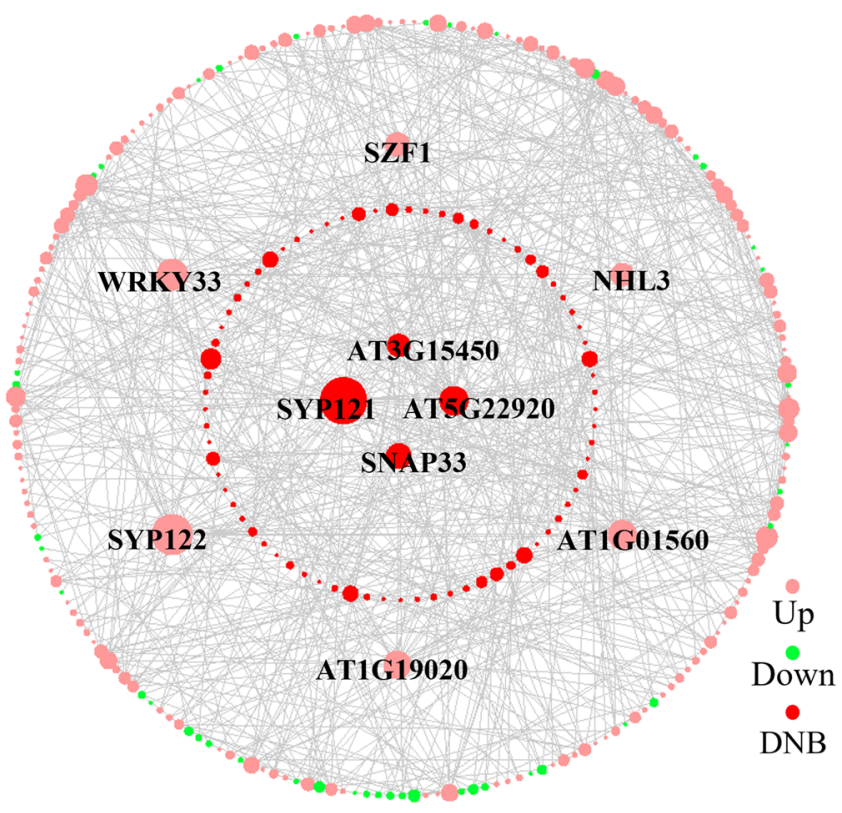

Fig. 5 The core network of DNB genes and DEG genes. a Volcano plot to display differential expressed genes between defense tipping point and previous time. $\mathbf{b}$ The core network between DNB genes and DEG genes. Nodes were sized according to connection degree. DNB genes were marked in red, up-regulated genes were marked in pink, and down-regulated genes were marked in green 
study also provided an information-rich resources to investigate JA signaling components in plant growth and defense [51].

Here, we provide a novel insight to identify the critical point and key genes of plant growth and defense transition response to JA. The DNB method identified the appearance of a group of genes with strongly collective fluctuations from the measured gene expression data to determine the critical transition of growth-to-defense in plants. Furthermore, the group members were predicted as the dynamic biomarkers for this critical transition. Based on the time-series high-throughput RNA-seq data of MeJA-treated Arabidopsis thaliana, we identified the critical growth-to-defense transition state and key genes by applying the non-modeled DNB approach.

The trade-offs of plant growth-defense have been studied and discussed a lot. These studies showed that the critical transition of growth-to-defense is key for plant defense. In the result of our study, the time point $8 \mathrm{~h}$ (8 $\mathrm{h}$ after MeJA treatment) was identified as the starting point of defense from minimizing growth and 146 genes were selected as DNB members. These genes detected by DNB extensively participate in the redistribution of metabolism which was involved in plant defense. For instance, activated by protein kinase complexes SnRK1, bZIP63 was considered as the central metabolic regulator essential for stress responses [40, 41]. The orthologs of bZIP63 were found in several plants regulating plant metabolism to pathogen defense [53, 54], and may participate in the signal crosstalk of ABA and auxin [41, 55]. ERD15 was identified as a central component in abiotic and biotic stress response in Arabidopsis thaliana [56]. Since the resources are limited, plants need to restrict their growth to ensure the defense for survival. Among the DNB genes, MYB3R5, TOPP9 and CDPK2 have been proved to be important negative regulators of plant growth. MYB3R5 inhibits plant growth by restricting the expression of the genes with mitotic functions [44]. While TOPP9 acts as a nucleocytoplasmic negative regulator of tip growth and affects the MAPKs-mediated defense [44].

Besides, several genes including AMT2, SYP121, CPK9, AtLEA5, AGB1 and SNAP33 in DNB members are not only involved in the 'jasmonic acid(JA)-mediated signaling' pathway, but also involved in other five pathways, i.e. 'salicylic acid mediated signaling pathway', 'response to the abscisic acid stimulus', 'signal transduction by protein phosphorylation', 'regulation of innate immune response', and 'defense response to fungus'. These genes may mediate phytohormone signaling crosstalk to regulate plant defense [3, 24, 57]. The function enrichment analysis revealed that DNB genes were widely involved in plant response to environment and plant signaling hormones, such as 'response to chitin', 'response to other organonitrogen compounds', 'regulation of innate immune response', 'abscisic acid-activated signaling pathway', etc. These results strongly supported that the DNB genes play an important role in plant growth-to-defense transition.

Furthermore, we analyzed the network of DNB genes and highlighted many hub genes including SYP121, SYP122, WRKY33, AT5G2290, MPK11, etc. These hub genes may perform key functions to plant growth defense. The top 1 hub gene SYP121 has been proved to promiscuously form SDS-resistant SNARE (soluble $N$ ethylmaleimide-sensitive factor protein attachment protein receptor) core complex together with the SNAP33 adaptor and a subset of VAMPs. Through the core complex, SYP121 drives vesicle traffic at the plasma membrane of cells throughout the vegetative plant [58, 59]. SYP121 plays a central role in plant growth and defense for that it not only facilitates targeted vesicle traffic for defense against pathogen attack to coordinate systemic immunity [20,60], but also participates in ABA pathway of cellular responses to drought and water stress $[59,61]$. The study of exogenous applications for labeled JAs indicated that JAs were able to be translocated to distal sites as signals $[62,63]$. Some studies suggest that JAs work together with system in the same signaling pathway that regulates systemic defense response with the mediation of SYP121 $[64,65]$. The top 2nd hub gene SYP122 (up-regulated) shares partial functional redundancy in vivo with the close homolog SYP121 with similar structures $[66,67]$.

In a previous research, the dynamic transcriptional regulation mechanism of JA gene regulatory network was studied [51]. The results showed that MeJA induces a burst of transcriptional activities which generate diverse expression patterns over time. In this study, we focus on the defense responses of plants induced by MeJA. With our special data analysis, we detected the tipping point of the growth-to-defense transition and identified a group of genes closely related to plant defense. The identified genes were proved to play a key role in plant defense and negative regulation of plant growth by previous studies $[44,68]$. The top genes identified were involved in regulating the redistribution of metabolism, plant responding to stress, and defensive signals transport. These identified genes provide candidates for engineering breeding that improves crop resistance or minimizes the consumption of resources to increase yields.

The DNB method can identify the critical transition points by analyzing the drastic fluctuations of a closely interacted molecular module in a system based on the high-throughput time-series data. Except for plant growth-defense transition, the DNB method can 
implement other situations such as disease or insect attacks if the perturbation data satisfying three criteria are available. At the situation of disease attacks, the DNB method has been proved to be effective for identifying the transition point of the disease during its progression [37]. To check whether MeJAinduced transition points are same every time, there should be some same experimental dataset for the analysis. If different systems are used for the comparison, the transition points of the systems can be identified but the key genes involved in the biological process are different because plants use different strategies to defense against different attacks [69].

In this work, we provide a new perspective for understanding the molecular mechanisms of growth-todefense transition. With a dynamic network analysis of MeJA-induced plant resistance, the tipping point of plant defense was detected. At the tipping point, the JA response genes in format of a module fluctuated drastically in expression. And a group of members were identified as the dynamic network biomarkers for this critical transition. The analysis showed that some of the identified genes have been widely proved by previous studies to play roles in plant defense and negative regulation in plant growth. Besides, we also provide a database with the key genes of plant defense induced by MeJA.

\section{Methods}

\section{Data collection and pre-processing}

The raw reads of high-resolution time-series RNA-seq dataset are accessible by number PRJNA224133 in the NCBI (National Center for Biotechnology Information) SRA (Short Read Archive) database (http://www.ncbi. nlm.nih.gov/sra/). The Arabidopsis was treated by MeJA as case and treated by mock as control. For each of the 13 time points $(0.25 \mathrm{~h}, 0.5 \mathrm{~h}, 1 \mathrm{~h}, 1.5 \mathrm{~h}, 2 \mathrm{~h}, 3 \mathrm{~h}, 4 \mathrm{~h}$, $5 \mathrm{~h}, 6 \mathrm{~h}, 7 \mathrm{~h}, 8 \mathrm{~h}, 9 \mathrm{~h}, 10 \mathrm{~h}, 12 \mathrm{~h}$ ) over a 12-hour period, 4 biological replicates and 4 technical replicates were performed. More details of the experiment can be found in the original report [70]. The raw reads were assessed with FastQC (v0.11.6; https://www.bioinformatics. babraham.ac.uk /projects/fastqc/) for quality control. HISAT (V2.1.0) was used to alignment the reads to the Arabidopsis thaliana reference genome(TAIR10) [71]. After alignment, StringTie (V1.3.3b) was used to assemble the aligned reads into full transcripts and to quantify Transcripts Per Kilobase of exon model per Million mapped reads (TPM) values of all genes. The gene expression level in each sample was calculated from the median value of four technical replicates for this sample. For further analysis, the genes with low abundance $(\mathrm{TPM}<1)$ were filtered out. The filtered gene expression data was listed in Supplementary Table S7.

\section{Differential expression analysis}

Differential gene expression analysis was performed using the R packge EBSeq (V0.36) which was developed based on an empirical Bayesian hierarchical model [72]. The genes with fold change $(\mathrm{FC})>1.5$ and false discovery rate $(\mathrm{FDR})<0.05$ were selected as DEGs.

\section{Function enrichment and visualization}

The GO and KEGG enrichment analysis for DNB genes were performed using $\mathrm{R}$ package topGO (V2.38.1, https://rdrr.io/bioc/topGO/) which was based on a hypergeometric test. The Arabidopsis GO (V3.10.0, https://bioconductor.org/packages/org.At.tair.db/) was chosen for the annotation analysis. The significant enrichment GO terms were chosen by the adjusted $P$ value $<0.05$. The dotplot and barplot were used for the visualization of the enriched GO terms.

\section{STRING network analysis}

To further analyze the function of DNB genes, the network between DNB and DEG genes was constructed with the STRING database as the background interactions. The parameter of comprehensive score was set to 0.6 for the filter of the network [73]. In the STRING network, the node represented the protein produced by a single and protein-coding gene locus and the edge represented protein-protein interactions (PPIs) including known and predicted interactions. The PPI score in the dataset indicated the confidence of interaction.

\section{Network visualization}

Cytoscape3.71 was used to visualize the network of gene interactions [74]. To survey the potential hub genes in growth-defense trends-off, the CytoHubba plug-in in Cytoscape was used to identify the hub genes.

\section{Abbreviations}

MeJA: Methyl jasmonate.; DNB: Dynamic network biomarker.; JA: Jasmonic acid.; JAZ: Jasmonate zim domain.; WGCNA: Weighted correlation network analysis.; SD: Standard deviation.; PCC: Pearson correlation coefficient.; Cl: Composite index.; PPI: Protein-protein interactions.; DEG: Differentially expressed genes.; GO: Gene Ontology.; KEGG: Kyoto Encyclopedia of Genes and Genomes.; TPM: Per million mapped reads.; FC: Fold change.; FDR: False discovery rate.

\section{Supplementary Information}

The online version contains supplementary material available at https://doi. org/10.1186/s12870-021-03185-1.

Additional file 1 : Supplementary Material. Figures for DNB module and $\mathrm{Cl}$ index.

Additional file 2 : Supplementary Table S1. List of DNB genes and their functional descriptions.

Additional file 3 : Supplementary Table S2. Functional enrichment for DNB genes.

Additional file 4 : Supplementary Table S3. List of DNB genes assigned KEGG pathways. 
Additional file 5 : Supplementary Table S4. List of differentially expressed genes between the defense tipping point and previous time point.

Additional file 6 : Supplementary Table S5. The network of DNB genes and DEGs

Additional file 7 : Supplementary Table S6. List of the top 10 hub genes of the core network of DNB genes and DEGs.

Additional file 8 : Supplementary Table S7. The list of filtered gene expression data.

\section{Acknowledgements}

We would like to thank the editor and reviewers' constructive comments and suggestions to improve the manuscript. We thank Richard Hickman and Marcel C. Van Verk from Department of Biology at Utrecht University for providing the time-series RNA-seq dataset of Arabidopsis thaliana treated by MeJA. We also thank Luonan Chen from Shanghai Institutes for Biological Sciences, Chinese Academy of Sciences for the suggestion on this project.

\section{Authors' contributions}

XZ conceived and designed the research; TW performed the research, analyzed the data and wrote the original draft. TW and XZ approved the final manuscript.

\section{Funding}

This work was supported by the National Natural Science Foundation of China [61402457, 32070682], Technology Innovation Zone Project [1716315XJ00200303, 1816315XJ00100216] and CAS Pioneer Hundred Talents Program.

\section{Availability of data and materials}

The raw RNA-seq data analyzed in this study are available in the NCBI SRA database, https://www.ncbi.nlm.nih.gov/sra/PRJNA224133 (accession BioProject number: PRJNA224133). The data supporting the findings of this study were included in the supplementary files.

\section{Declarations}

Ethics approval and consent to participate

We declare that the study complied with relevant institutional, national, and international guidelines and legislation for using Arabidopsis thaliana.

\section{Consent for publication}

Not applicable.

\section{Competing interests}

The authors declare that they have no competing interests.

\section{Author details}

'CAS Key Laboratory of Plant Germplasm Enhancement and Specialty Agriculture, Wuhan Botanical Garden, Chinese Academy of Sciences, 430074 Wuhan, China. ${ }^{2}$ Center of Economic Botany, Core Botanical Gardens, Chinese Academy of Sciences, 430074 Wuhan, China. ${ }^{3}$ University of Chinese Academy of Sciences, 100049 Beijing, China.

Received: 5 February 2021 Accepted: 23 August 2021

Published online: 06 October 2021

\section{References}

1. Huot B, Yao J, Montgomery BL, He SY. Growth-defense tradeoffs in plants: a balancing act to optimize fitness. Mol Plant. 2014;7(8):1267-87.

2. Campos ML, Yoshida Y, Major IT, de Oliveira Ferreira D, Weraduwage SM, Froehlich JE, Johnson BF, Kramer DM, Jander G, Sharkey TD, et al. Rewiring of jasmonate and phytochrome B signalling uncouples plant growthdefense tradeoffs. Nat Commun. 2016:7:12570.

3. Guo Q, Major IT, Howe GA. Resolution of growth-defense conflict: mechanistic insights from jasmonate signaling. Curr Opin Plant Biol. 2018;44: 72-81.

4. Coley PD, Bryant JP, Chapin FS. 3rd: Resource availability and plant antiherbivore defense Science. 1985;230(4728):895-9.
5. Wang W, Wang Z-Y. At the intersection of plant growth and immunity. Cell Host Microbe. 2014;15(4):400-2.

6. Wasternack C. Sulfation switch in the shade. Nature Plants. 2020;6(3):186-7.

7. Ishihama N, Yamada R, Yoshioka M, Katou S, Yoshioka H. Phosphorylation of the Nicotiana benthamiana WRKY8 transcription factor by MAPK functions in the defense response. Plant Cell. 2011;23(3):1153.

8. Howe GA, Major IT, Koo AJ. Modularity in jasmonate signaling for multistress resilience. Annu Rev Plant Biol. 2018;69:387-415.

9. Dempsey DA, Klessig DF. SOS - too many signals for systemic acquired resistance? Trends Plant Sci. 2012;17(9):538-45.

10. Mine A, Seyfferth C, Kracher B, Berens ML, Becker D, Tsuda K. The defense phytohormone signaling network enables rapid, high-amplitude transcriptional reprogramming during effector-triggered immunity. Plant Cell. 2018;30(6):1199-219.

11. Naseem M, Philippi N, Hussain A, Wangorsch G, Ahmed N, Dandekar T. Integrated systems view on networking by hormones in Arabidopsis immunity reveals multiple crosstalk for cytokinin. Plant Cell. 2012;24(5):1793814.

12. Kazan K. Diverse roles of jasmonates and ethylene in abiotic stress tolerance. Trends Plant Sci. 2015;20(4):219-29.

13. Wilkinson S, Kudoyarova GR, Veselov DS, Arkhipova TN, Davies WJ. Plant hormone interactions: innovative targets for crop breeding and management. J Exp Bot. 2012;63(9):3499-509.

14. Cheong JJ, Choi YD. Methyl jasmonate as a vital substance in plants. Trends Genet. 2003;19(7):409-13.

15. Ahmad P, Rasool S, Gul A, Sheikh SA, Akram NA, Ashraf M, Kazi AM, Gucel S. Jasmonates: multifunctional roles in stress tolerance. Front Plant Sci. 2016;7: 813.

16. Pieterse CMJ, Does DVd, Zamioudis C, Leon-Reyes A, Wees SCMV. Hormonal modulation of plant immunity. Annu Rev Cell Dev Biol. 2012;28(1):489-521.

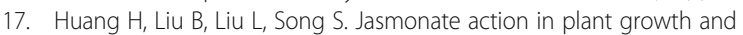
development. J Exp Bot. 2017:68(6):1349-59.

18. Withers J, Yao J, Mecey C, Howe GA, Melotto M, He SY. Transcription factordependent nuclear localization of a transcriptional repressor in jasmonate hormone signaling. Proc Natl Acad Sci. 2012;109(49):20148-53.

19. Browse J. Jasmonate passes muster: a receptor and targets for the defense hormone. Annu Rev Plant Biol. 2009;60(1):183-205.

20. Zhang $H$, Zhang $H$, Lin J. Systemin-mediated long-distance systemic defense responses. New Phytol. 2020;226(6):1573-82.

21. Zeng L, Tu XL, Dai H, Han FM, Lu BS, Wang MS, Nanaei HA, Tajabadipour A, Mansouri $\mathrm{M}$, Li XL, et al. Whole genomes and transcriptomes reveal adaptation and domestication of pistachio. Genome Biol. 2019;20(1):79.

22. Per TS, Khan MIR, Anjum NA, Masood A, Hussain SJ, Khan NA. Jasmonates in plants under abiotic stresses: crosstalk with other phytohormones matters. Environ Exp Bot. 2018;145:104-20

23. Yang J, Duan G, Li C, Liu L, Han G, Zhang Y, Wang C. The Crosstalks Between Jasmonic Acid and Other Plant Hormone Signaling Highlight the Involvement of Jasmonic Acid as a Core Component in Plant Response to Biotic and Abiotic Stresses. Front Plant Sci. 2019:10:1349.

24. Savchenko TV, Rolletschek $H$, Dehesh $\mathrm{K}$. Jasmonates-mediated rewiring of central metabolism regulates adaptive responses. Plant Cell Physiol. 2019; 60(12):2613-20.

25. Trang Nguyen $\mathrm{H}$, Thi Mai To H, Lebrun M, Bellafiore S, Champion A. Jasmonates-the master regulator of rice development, adaptation and defense. Plants (Basel). 2019:8(9):339.

26. Sheard LB, Tan X, Mao H, Withers J, Ben-Nissan G, Hinds TR, Kobayashi Y, Hsu F-F, Sharon M, Browse J, et al. Jasmonate perception by inositolphosphate-potentiated COI1-JAZ co-receptor. Nature. 2010;468(7322): 400-5.

27. Zhao J, Zhou Y, Zhang $X$, Chen L. Part mutual information for quantifying direct associations in networks. Proc Natl Acad Sci. 2016;113(18):5130.

28. Zhang XJ, Liu KQ, Liu ZP, Duval B, Richer JM, Zhao XM, Hao JK, Chen LN NARROMI: a noise and redundancy reduction technique improves accuracy of gene regulatory network inference. Bioinformatics (Oxford, England). 2013;29(1):106-13.

29. Zhang X, Zhao J, Hao JK, Zhao XM, Chen L. Conditional mutual inclusive information enables accurate quantification of associations in gene regulatory networks. Nucleic Acids Res. 2015:43(5):e31.

30. Zheng G, Xu Y, Zhang X, Liu ZP, Wang Z, Chen L, Zhu XG. CMIP: a software package capable of reconstructing genome-wide regulatory networks using gene expression data. BMC Bioinformatics. 2016;17(17):535. 
31. Langfelder P, Horvath S. WGCNA: an R package for weighted correlation network analysis. BMC Bioinformatics. 2008;9:559.

32. Zhang N, Zhao B, Fan Z, Yang D, Guo X, Wu Q, Yu B, Zhou S, Wang H. Systematic identification of genes associated with plant growth-defense tradeoffs under JA signaling in Arabidopsis. Planta. 2020;251(2):43.

33. Zhang W, Corwin JA, Copeland D, Feusier J, Eshbaugh R, Chen F, Atwell S, Kliebenstein DJ. Plastic transcriptomes stabilize immunity to pathogen diversity: the jasmonic acid and salicylic acid networks within the Arabidopsis Botrytis pathosystem. Plant Cell. 2017;29(11):2727-52.

34. Li M, Zeng T, Liu R, Chen L. Detecting tissue-specific early warning signals for complex diseases based on dynamical network biomarkers: study of type 2 diabetes by cross-tissue analysis. Brief Bioinform. 2013; 15(2):229-43.

35. Liu R, Wang J, Ukai M, Sewon K, Chen P, Suzuki Y, Wang H, Aihara K, OkadaHatakeyama M, Chen L. Hunt for the tipping point during endocrine resistance process in breast cancer by dynamic network biomarkers. J Mol Cell Biol. 2019;11(8):649-64.

36. Zhang FP, Liu XP, Zhang AD, Jiang ZL, Chen LN, Zhang XJ. Genome-wide dynamic network analysis reveals a critical transition state of flower development in Arabidopsis. BMC Plant Biol. 2019;19:18.

37. Yang B, Li M, Tang W, Liu W, Zhang S, Chen L, Xia J. Dynamic network biomarker indicates pulmonary metastasis at the tipping point of hepatocellular carcinoma. Nat Commun. 2018;9(1):678.

38. Roy S. Time-intensive transcriptomics reveal temporal patterns in the jasmonic acid gene regulatory network. Plant Cell. 2017;29(9):2078-9.

39. Bechtold U, Penfold CA, Jenkins DJ, Legaie R, Moore JD, Lawson T, Matthews JSA, Vialet-Chabrand SRM, Baxter L, Subramaniam S, et al. Timeseries transcriptomics reveals that AGAMOUS-LIKE22 affects primary metabolism and developmental processes in drought-stressed arabidopsis. Plant Cell. 2016;28(2):345-66.

40. Mair A, Pedrotti L, Wurzinger B, Anrather D, Simeunovic A, Weiste C, Valerio C, Dietrich K, Kirchler T, Nägele T, et al. SnRK1-triggered switch of bZIP63 dimerization mediates the low-energy response in plants. ELife. 2015;4: e05828.

41. Matiolli CC, Tomaz JP, Duarte GT, Prado FM, Del Bem LE, Silveira AB, Gauer L, Corrêa LG, Drumond RD, Viana AJ, et al. The Arabidopsis bZIP gene AtbZIP63 is a sensitive integrator of transient abscisic acid and glucose signals. Plant Physiol. 2011;157(2):692-705.

42. Liu T, Longhurst AD, Talavera-Rauh F, Hokin SA, Barton MK. The Arabidopsis transcription factor $A B I G 1$ relays $A B A$ signaled growth inhibition and drought induced senescence. Elife. 2016;5:e13768.

43. Kobayashi K, Suzuki T, Iwata E, Nakamichi N, Suzuki T, Chen P, Ohtani M, Ishida T, Hosoya H, Müller S, et al. Transcriptional repression by MYB3R proteins regulates plant organ growth. Embo J. 2015;34(15):1992-2007.

44. Liu Y, Yan J, Qin Q, Zhang J, Chen Y, Zhao L, He K, Hou S. Type one protein phosphatases (TOPPs) contribute to the plant defense response in Arabidopsis. J Integr Plant Biol. 2020;62(3):360-77.

45. Kanehisa M, Sato Y, Kawashima M, Furumichi M, Tanabe M. KEGG as a reference resource for gene and protein annotation. Nucleic Acids Res. 2016;44(D1):D457-462.

46. Zhang J, Li X-M, Lin H-X, Chong K. Crop improvement through temperature resilience. Annu Rev Plant Biol. 2019;70(1):753-80.

47. Brown JKM. Yield penalties of disease resistance in crops. Curr Opin Plant Biol. 2002;5(4):339-44.

48. Ning Y, Liu W, Wang G-L. Balancing immunity and yield in crop plants. Trends Plant Sci. 2017;22(12):1069-79.

49. Guo Q, Major IT, Howe GA. Resolution of growth-defense conflict: mechanistic insights from jasmonate signaling. Curr Opin Plant Biol. 2018;44 72-81.

50. Zander M, Lewsey MG, Clark NM, Yin L, Bartlett A, Saldierna Guzmán JP, Hann E, Langford AE, Jow B, Wise A, et al. Integrated multi-omics framework of the plant response to jasmonic acid. Nature Plants. 2020; 6(3):290-302.

51. Hickman R, Van Verk MC, Van Dijken AJH, Mendes MP, Vroegop-Vos IA, Caarls L, Steenbergen M, Van der Nagel I, Wesselink GJ, Jironkin A, et al. Architecture and dynamics of the jasmonic acid gene regulatory network. Plant Cell. 2017;29(9):2086-105.

52. Aerts N, Pereira Mendes M, Van Wees SCM. Multiple levels of crosstalk in hormone networks regulating plant defense. Plant J. 2021;105(2):489-504.

53. Dröge-Laser $W$, Weiste $C$. The C/S1 bZIP network: a regulatory hub orchestrating plant energy homeostasis. Trends Plant Sci. 2018;23(5):422-33.
54. Shen L, Liu Z, Yang S, Yang T, Liang J, Wen J, Liu Y, Li J, Shi L, Tang Q, et al. Pepper CabZIP63 acts as a positive regulator during Ralstonia solanacearum or high temperature-high humidity challenge in a positive feedback loop with CaWRKY40. J Exp Bot. 2016;67(8):2439-51.

55. Kuhlmann M, Horvay K, Strathmann A, Heinekamp T, Fischer U, Böttner S, Dröge-Laser W. The a-helical D1 domain of the tobacco bZIP transcription factor BZl-1 interacts with the ankyrin-repeat protein ANK1 and is important for BZI-1 function, both in auxin signaling and pathogen response. J Biol Chem. 2003;278(10):8786-94.

56. Aalto MK, Helenius E, Kariola T, Pennanen V, Heino P, Hõrak H, Puzõrjova I, Kollist H, Palva ET. ERD15-An attenuator of plant ABA responses and stomatal aperture. Plant Sci. 2012;182:19-28.

57. Berens ML, Berry HM, Mine A, Argueso CT, Tsuda K. Evolution of hormone signaling networks in plant defense. Annu Rev Phytopathol. 2017;55:401-25.

58. Kwon C, Neu C, Pajonk S, Yun HS, Lipka U, Humphry M, Bau S, Straus M, Kwaaitaal M, Rampelt $\mathrm{H}$, et al. Co-option of a default secretory pathway for plant immune responses. Nature. 2008;451(7180):835-40.

59. Karnik R, Grefen C, Bayne R, Honsbein A, Köhler T, Kioumourtzoglou D, Williams M, Bryant NJ, Blatt MR. Arabidopsis Sec1/Munc18 protein SEC11 is a competitive and dynamic modulator of SNARE binding and SYP121dependent vesicle traffic. Plant Cell. 2013;25(4):1368-82.

60. Wang L, Einig E, Almeida-Trapp M, Albert M, Fliegmann J, Mithöfer A, Kalbacher H, Felix G. The systemin receptor SYR1 enhances resistance of tomato against herbivorous insects. Nat Plants. 2018;4(3):152-6.

61. Collins NC, Thordal-Christensen H, Lipka V, Bau S, Kombrink E, Qiu JL, Hückelhoven R, Stein M, Freialdenhoven A, Somerville SC, et al. SNAREprotein-mediated disease resistance at the plant cell wall. Nature. 2003; 425(6961):973-7.

62. Jimenez-Aleman GH, Scholz SS, Heyer M, Reichelt M, Mithöfer A, Boland W. Synthesis, metabolism and systemic transport of a fluorinated mimic of the endogenous jasmonate precursor OPC-8:0. Biochimica et Biophysica Acta (BBA) - Molecular and Cell Biology of Lipids. 2015; 1851(12):1545-53

63. Thorpe MR, Ferrieri AP, Herth MM, Ferrieri RA. 11C-imaging: Methyl jasmonate moves in both phloem and xylem, promotes transport of jasmonate, and of photoassimilate even after proton transport is decoupled. Planta. 2007;226(2):541-51.

64. Sun JQ, Jiang HL, Li CY. Systemin/Jasmonate-mediated systemic defense signaling in tomato. Mol Plant. 2011;4(4):607-15.

65. Kandoth PK, Ranf S, Pancholi SS, Jayanty S, Walla MD, Miller W, Howe GA, Lincoln DE, Stratmann JW. Tomato MAPKs LeMPK1, LeMPK2, and LeMPK3 function in the systemin-mediated defense response against herbivorous insects. Proc Natl Acad Sci U S A. 2007;104(29):12205-10.

66. Grefen C, Chen Z, Honsbein A, Donald N, Hills A, Blatt MR. A Novel Motif Essential for SNARE Interaction with the K+ Channel KC1 and Channel Gating in Arabidopsis. Plant Cell. 2010;22(9):3076-92.

67. Zhang B, Karnik R, Alvim J, Donald N, Blatt MR. Dual Sites for SEC11 on the SNARE SYP121 Implicate a Binding Exchange during Secretory Traffic. Plant Physiol. 2019;180(1):228-39.

68. Franck CM, Westermann J, Bürssner S, Lentz R, Lituiev DS, Boisson-Dernier A. The protein phosphatases ATUNIS1 and ATUNIS2 regulate cell wall integrity in tip-growing cells. Plant Cell. 2018;30(8):1906-23.

69. Antico CJ, Colon C, Banks T, Ramonell KM. Insights into the role of jasmonic acid-mediated defenses against necrotrophic and biotrophic fungal pathogens. Front Biol. 2012;7(001):48-56.

70. Hickman R, Van Verk MC, Van Dijken AJH, Mendes MP, Vroegop-Vos IA, Caarls L, Steenbergen M, Van der Nagel I, Wesselink GJ, Jironkin A, et al. Architecture and dynamics of the jasmonic acid gene regulatory network. Plant Cell. 2017;29(9):2086.

71. Pertea M, Kim D, Pertea GM, Leek JT, Salzberg SL. Transcript-level expression analysis of RNA-seq experiments with HISAT, StringTie and Ballgown. Nat Protoc. 2016;11(9):1650-67.

72. Leng N, Dawson JA, Thomson JA, Ruotti V, Rissman Al, Smits BMG, Haag JD, Gould MN, Stewart RM, Kendziorski C. EBSeq: an empirical Bayes hierarchical model for inference in RNA-seq experiments. Bioinformatics (Oxford, England). 2013;29(8):1035-43.

73. Szklarczyk D, Gable AL, Lyon D, Junge A, Wyder S, Huerta-Cepas J, Simonovic M, Doncheva NT, Morris JH, Bork P, et al. STRING V11: protein-protein association networks with increased coverage, supporting functional discovery in genome-wide experimental datasets. Nucleic Acids Res. 2019;47(D1):D607-d613. 
74. Shannon P, Markiel A, Ozier O, Baliga NS, Wang JT, Ramage D, Amin N, Schwikowski B, Ideker T. Cytoscape: a software environment for integrated models of biomolecular interaction networks. Genome Res. 2003;13(11): 2498-504.

\section{Publisher's Note}

Springer Nature remains neutral with regard to jurisdictional claims in published maps and institutional affiliations.

Ready to submit your research? Choose BMC and benefit from:

- fast, convenient online submission

- thorough peer review by experienced researchers in your field

- rapid publication on acceptance

- support for research data, including large and complex data types

- gold Open Access which fosters wider collaboration and increased citations

- maximum visibility for your research: over $100 \mathrm{M}$ website views per year

At $\mathrm{BMC}$, research is always in progress.

Learn more biomedcentral.com/submissions 